\title{
Stage-specific requirement of kinase PDK1 for NK cells development and activation
}

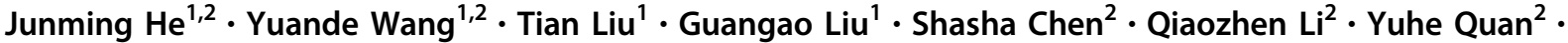 \\ Haoyan Yang ${ }^{2} \cdot$ Jin Feng ${ }^{2} \cdot$ Song Wang $^{3} \cdot$ Meixiang Yang ${ }^{1} \cdot$ Zhongjun Dong $^{2}$
}

Received: 18 August 2018 / Revised: 16 November 2018 / Accepted: 5 December 2018 / Published online: 8 January 2019

(c) ADMC Associazione Differenziamento e Morte Cellulare 2019

\begin{abstract}
Phosphoinositide-dependent kinase-1 (PDK1) is an important enzyme for immune cell development by connecting PI3K to downstream mTOR signaling. It is needed to investigate how PDK1 spatiotemporally orchestrates NK cells development and whether this kinase is required for NK cells effector function. In this study, we used three genetic models to delete $p d k 1$ at respective developmental stages, including hematopoietic stem cells (Vav1-Cre used), NK cell progenitor (NKp, CD122Cre used) and terminal NK cells (Ncr1-Cre used). We found that CD122-Cre mediated deletion of $p d k 1$ caused a severe loss of NK cells to an extent comparable to that of deletion by Vav1-Cre, and further revealed that PDK1 was necessary for NK cells master transcription factor E4BP4 expression at the NKp stage. Moreover, Ncr1-Cre-mediated inactivation of $p d k 1$ delayed NK cells terminal differentiation. These PDK1-deficient NK cells secreted decreased amounts of the cytokine IFN- $\gamma$, likely due to impaired downstream mTOR activation. They also exhibited reduced degranulation in response to tumor cells. Mechanistically, PDK1 was critical for the formation of NK-target conjugates and lytic synapses. Therefore, we clarify the stage-specific roles of the metabolic regulator PDK1 in NK cells biology.
\end{abstract}

\section{Introduction}

Natural killer (NK) cells are innate lymphocytes capable of mediating cytotoxicity against tumor cells and virus-

\section{Edited by H-U Simon}

Supplementary information The online version of this article (https:// doi.org/10.1038/s41418-018-0263-8) contains supplementary material, which is available to authorized users.

Meixiang Yang

yangmxqilu@163.com

$\triangle$ Zhongjun Dong

dongzj@biomed.tsinghua.edu.cn

1 The First Affiliated Hospital, Biomedical Translational Research Institute, Guangdong Province Key Laboratory of Molecular Immunology and Antibody Engineering, Jinan University, Guangzhou, China

2 School of Medicine and Institute for Immunology, Beijing Key Lab for Immunological Research on Chronic Diseases, Tsinghua University, Beijing 100084, China

3 Exercise Immunology Center, Wuhan Sports University, Wuhan 340036, China infected cells, as well as rejecting major histocompatibility complex class I (MHC-I)-mismatched allogeneic bone marrow [1]. NK cells exert their effector functions through secretion of cytolytic granules and cytokines, which is modulated by the coordinated interaction of a vast variety of cell surface receptors [2]. These receptors are classified into inhibitory and activating receptors. Inhibitory receptors, such as inhibitory Ly49s in mice and killer inhibitory receptors in humans, recognize the normal expression of host cellular proteins especially MHC-I molecules, thereby avoiding NK cell destruction of healthy cells [3]. The inhibitory receptors Siglec-7 and 9 on human NK cells also recognize sialic acid-containing carbohydrates [4]. On the other hand, activating receptors, such as NKG2D and Ly49H, sense pan-specific ligands induced by cellular stress or viral infection. Engagement of these activating receptors with their ligands on target cells induces receptor clustering at the interface, and can activate NK cells to produce cytokines and cytolytic effector molecules to kill target cells $[5,6]$. Due to the lack of cytoplasmic signaling elements in these receptors, delivery of signals is transmitted through other adaptor proteins, such as DAP10, DAP12, and FcR $\gamma$, depending on the type of engaged receptors $[5,7]$. Therefore, the NK cell effector response is often determined by 
the integration of signal transduction pathways, even though they were originally recognized as "ready to act" cells. How distinct NK cell receptors and downstream signaling selectively mediate cytotoxicity and cytokine production remains an active research area.

NK cells also express receptors for multiple soluble cytokines. IL-15 is the most important cytokine among them in enabling NK cells homeostasis, maturation, and activation [8-10]. Engagement of the IL-15 receptor on NK cells causes autophosphorylation and activation of phosphatidylinositol-4, 5-bisphosphate 3-kinase (PI3K) and mammalian target of rapamycin (mTOR) pathway $[11,12]$. Mice simultaneously lacking the PI3K subunits P110 $\gamma$ and $\delta$ exhibit a severe defect in early NK cell development and function [13, 14]. Ablation of mTOR affects NK cell blastogenesis, activation, and effector functions [15].

Phosphoinositide-dependent kinase-1 (PDK1) has been considered as a critical regulator connecting PI3K and components downstream of mTOR activation. An important role for PDK1 is to phosphorylate the T308 site of AKT and synergize with mTORC2 to fully activate downstream AKT. PDK1 has been shown to play a critical role in the development of $\mathrm{T}$ cells and B cells [16-18]. Recently, we observed that deficiency of PDK1 caused almost a 95\% reduction in NK cells number [19]. However, how PDK1 spatiotemporally orchestrates NK cells development and whether this kinase is required for NK cells effector function needs to be further investigated. In this study, we generated three lines of conditional PDK1 knockout mice, in which the $p d k 1$ gene was deleted at various developmental stages. Our results reveal that PDK1 plays multifaceted roles in the regulation of NK cells commitment and activation. These findings establish PDK1 as a critical member of the PI $3 \mathrm{~K}$ signaling network that governs early NK cells development and peripheral immune functions.

\section{Results}

\section{Deletion of PDK1 at the NKp stage severely blocks NK cells development}

Mature NK (mNK) cells are derived from hematopoietic stem cells (HSCs) through multiple developmental stages, including common lymphoid progenitor, pre-NK progenitor (NKp), immature NK, and mNK cells [20]. We previously found that the loss of PDK1 at the HSC stage in PDKI $\mathrm{flfl} /$ Vav1-Cre ${ }^{+}$mice (hereafter referred to as PDK1 $1^{\mathrm{Vavl}-\mathrm{Cre}}$ ) caused a severe defect in NK cells development [19]. To understand in-depth how this kinase dictates NK cells development at a spatiotemporal level, we first quantified precursors of NKp cells, including HSCs, CLPs, and preNKp cells. We found that the relative proportions of these three populations in PDK1 ${ }^{\text {Vav1-Cre }}$ mice were nearly comparable with those in wild-type (WT) mice (Fig. 1a-c). This result implies that PDK1 is dispensable for the ontogeny of the earliest NK progenitors, at least before the NKp stage. Notably, there were pronouncedly fewer NKp cells in PDK $1^{\text {Vav1-Cre }}$ bone marrow $(\mathrm{BM})$, suggesting a role for PDK1 in NK cell commitment. (Fig. 1b, c).

In an effort to confirm this role, we generated a novel PDK1-deficient model, $P D K 1^{f / f} / C D 122-C r e^{+}$(hereafter referred to as $\mathrm{PDK} 1^{\mathrm{CD} 122-\mathrm{Cre}}$ ), in which $p d k 1$ is deleted at the NKp stage. CD122-Cre mediated PDK1 depletion did not affect the number of $\mathrm{T}$ and $\mathrm{B}$ cells in the evaluated tissues (Fig. 1d, e). As expected, these mice had a significant reduction of NK-T cell percentages and absolute numbers in the spleen and liver (Fig. 1f, g). These data suggest that CD122-Cre-mediated PDK1 deletion does not disturb B cell-lineage and conventional $\mathrm{T}$ cells. We next performed a thorough analysis of NK cells development in this genotype. PDK1 ${ }^{\mathrm{CD} 122-\mathrm{Cre}}$ mice had a nearly $95 \%$ reduction in the number of NK cells of the spleen, BM, liver, lungs, and lymph nodes, compared to those from the WT mice (Fig. 1h, i). Thus, the deletion of PDK1 at the NKp stage severely blocks NK cells development.

To strengthen this notion, we examined the subsets of residual NK cells in PDK1 ${ }^{\mathrm{CD} 122-\mathrm{Cre}}$ mice according to 4-stage taxonomy [21]. In PDK1 ${ }^{\mathrm{CD} 122-\mathrm{Cre}}$ mice, two NK cells subpopulations, $\mathrm{CD} 27^{-} \mathrm{CD} 11 \mathrm{~b}^{-}$and $\mathrm{CD} 27^{+} \mathrm{CD} 11 \mathrm{~b}^{-}$, which represent immature NK cells, were dramatically enriched; however, the proportion of $\mathrm{mNK}$ cells that were $\mathrm{CD} 27^{-}$ $\mathrm{CD}_{1} 1 \mathrm{~b}^{+}$was significantly reduced (Fig. $1 \mathrm{j}, \mathrm{k}$ ). Over the course of their maturation, NK cells must sequentially acquire various receptors as a consequence of their development and function. In-depth analysis of these markers demonstrated that the proportion of NK cells expressing Ly49 family members was minimal in the spleen of PDK1 ${ }^{\mathrm{CD} 122-\mathrm{Cre}}$ mice, whereas the proportion of NK cells with immature markers, either CD117 or CD127, was much higher (Fig. 11). These data collectively suggest that PDK1 is most likely required for NK cells development at the NKp stage.

Considering that innate lymphoid cell (ILC) 1 also expresses a high amount of CD122 [22], we then examined the development of ILC1 $\left(\mathrm{CD} 3^{-} \mathrm{NK} 1.1^{+} \mathrm{CD} 49 \mathrm{a}^{+} \mathrm{CD} 49 \mathrm{~b}^{-}\right)$ in PDK1 $1^{\mathrm{CD} 122-\mathrm{Cre}}$ mice. Notably, the absolute numbers of ILC1 were significantly attenuated in the BM and liver of PDK1-deleted mice, implying that PDK1 is also required for the generation of ILC1 (Fig. 1m).

\section{Deletion of PDK1 at the NKp stage severely compromises NK-mediated surveillance}

NK cells play a central role in rejecting allogeneic bone marrow cells and tumors. We first measured the ability of NK cells function ex vivo. The residual NK cells in 

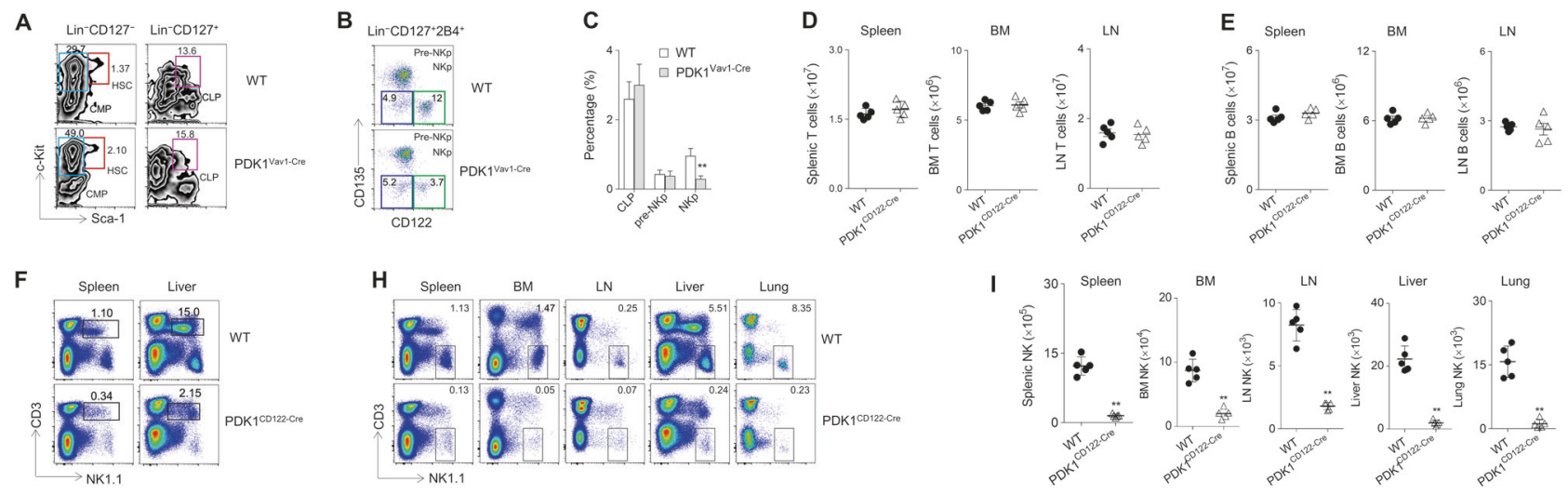

G
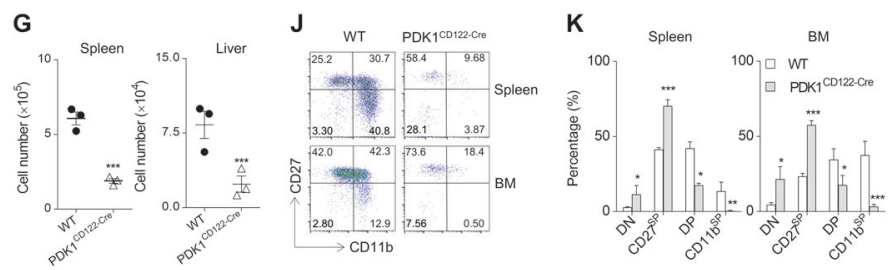

L

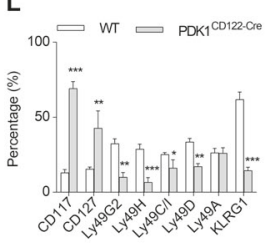

M

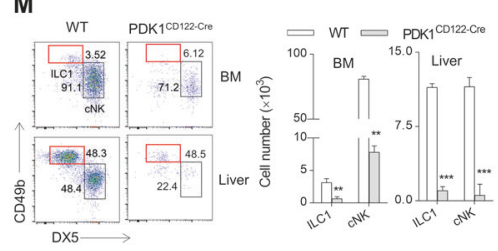

Fig. 1 CD122-Cre mediated $p d k 1$ deletion severely compromises NK cells development. a-c Representative flow cytometry plots $(\mathbf{a}, \mathbf{b})$ and quantification (c) of hematopoietic stem cells (HSC, $\mathrm{Lin}^{-} \mathrm{CD} 127^{-}$ c-Kit ${ }^{+} \mathrm{Sca}-1^{+}$), common myeloid progenitors (CMP, $\mathrm{Lin}^{-} \mathrm{CD} 127^{-}$ c-Kit ${ }^{+} \mathrm{Sca}-1^{-}$) and common lymphoid progenitors (CLP, $\mathrm{Lin}^{-} \mathrm{CD} 127^{+}$

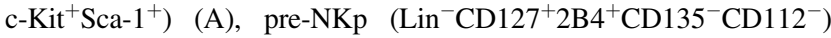
and $\mathrm{NKp}\left(\mathrm{Lin}^{-} \mathrm{CD} 127^{+} 2 \mathrm{~B} 4^{+} \mathrm{CD} 135^{-} \mathrm{CD} 112^{+}\right)(\mathbf{b})$ in the BM of WT and PDK $1^{\text {Vav1-Cre }}$ mice. Numbers near the indicated square box show the respective percentage. $\mathbf{d}$, $\mathbf{e}$ The absolute number of $\mathrm{T}$ cells $(\mathbf{d})$ and $\mathrm{B}$ cells (e) in the indicated tissues and organs from WT and PDK1 ${ }^{\mathrm{CD} 122-\mathrm{Cre}}$ mice. f, $\mathbf{g}$ Representative flow cytometric profiles (f) and the absolute number $(\mathrm{g})$ of NK-T cells $\left(\mathrm{CD} 3^{\text {low }} \mathrm{NK} 1.1^{\text {low }}\right)$ in the

PDK1 ${ }^{\mathrm{CD} 122-\mathrm{Cr}}$ mice exhibited a severe defect in IFN- $\gamma$ production and CD107a expression following stimulation with MHC-I-missing RMA-S or YAC-1 cells (Fig. 2a, b). Consistently, minimal NK cells activity of in vivo rejection of $\mathrm{MHC}^{-\mathrm{I}^{-l-}}$ splenocytes was detectable in PDK1 ${ }^{\mathrm{CD} 122-\mathrm{Cre}}$ mice (Fig. 2c). NK cells in PDK1 ${ }^{\mathrm{CD} 122-\mathrm{Cre}}$ mice also hardly removed RMA-S cells in the peritoneal cavity (Fig. 2d). Finally, we found that both lung weight and numbers of tumor colonies in PDK $1^{\mathrm{CD} 122-\mathrm{Cre}}$ mice were dramatically greater than those in control WT mice (Fig. 2e). As expected, NK cells were rarely detectable in the lung of

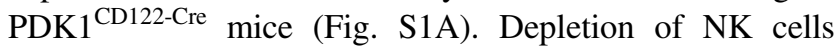
using anti-NK1.1 antibody significantly reduced the difference between two genotypes (Fig. 2e), suggesting that the control of B16 lung metastasis is NK cells dependent. Thus, deletion of PDK1 at the NKp stage compromised NK cells immunosurveillance.

\section{PDK1 regulates NK cells commitment by inducing E4BP4}

We next evaluated whether deletion of PDK1 at the NKp stage might disturb PI3K-mTOR signal transduction. Notably, spleens and livers of WT and PDK1 $1^{\mathrm{CD} 122-\mathrm{Cre}}$ mice. $\mathbf{h}, \mathbf{i}$ Representative flow cytometric profiles (h) and the absolute number (i) of NK cells $\left(\mathrm{CD}^{-} \mathrm{NK} 1.1^{+}\right)$in the spleen, BM, LN, liver, and lungs of WT and PDK1 ${ }^{\mathrm{CD} 122-C r e}$ mice. $\mathbf{j}, \mathbf{k}$ Representative flow cytometric profiles (j) and the percentages $(\mathbf{k})$ of NK cell subsets in the spleen and BM from the WT and PDK1 $1^{\mathrm{CD} 122-\mathrm{Cre}}$ mice. DN $\left(\mathrm{CD} 27^{-} \mathrm{CD} 11 \mathrm{~b}^{-}\right), \mathrm{CD} 27 \mathrm{SP}$ $\left(\mathrm{CD} 27^{+} \mathrm{CD} 11 \mathrm{~b}\right)$, DP $\left(\mathrm{CD} 27^{+} \mathrm{CD} 11 \mathrm{~b}^{+}\right)$and CD11b SP $\left(\mathrm{CD} 27^{-} \mathrm{CD} 11 \mathrm{~b}\right.$ $\left.{ }^{+}\right)$cells. I Percentage of developmental markers on splenic NK cells $\left(\mathrm{CD}^{-} \mathrm{NK} 1.1^{+}\right)$in WT and PDK1 ${ }^{\mathrm{CD} 122-\mathrm{Cre}}$ mice. m Representative flow cytometry plots and quantification of BM and liver ILC1 (CD3 ${ }^{-} \mathrm{NK} 1.1^{+} \mathrm{CD} 49 \mathrm{a}^{+} \mathrm{CD} 49 \mathrm{~b}^{-}$). The data represent one of three independent experiments, and values are expressed as the mean \pm s.d

phosphorylation of S6, representing mTOR activation, was significantly decreased in PDK1-deleted NK cells upon IL15/IL-15Ra complex (IL-15C) stimulation. Threonine phosphorylation at the site 308 of AKT, was also minimal in PDK1 ${ }^{\text {CD122-Cre }}$ NK cells (Fig. 3a, b). Thus, the disruption of PDK1 in the NKp stage disconnects IL-15-triggered mTOR activation. NK cells development highly depends on the transcription factor E4BP4 [23, 24]. We previously showed that loss of PDK1 at the HSC stage could inhibit the IL-15induced expression of E4BP4 in NK cells. To further investigate the mechanism of how PDK1 regulates NKp differentiation, we examined the expression levels of E4BP4 in PDK1 ${ }^{\mathrm{CD} 122-\mathrm{Cre}}$ mice. The IL-15C-stimulated NK cells from PDK1 ${ }^{\mathrm{CD} 122-\mathrm{Cre}}$ mice exhibited defective expression of E4BP4 and two T-box transcription factors, Eomes and T-bet (Fig. 3c-f). Thus, these data further demonstrate that PDK1 regulates NK cells development, most likely at the NKp stage.

\section{Fate mapping reveals that PDK1 is required for terminal NK cells differentiation}

IL-15 signaling is not only essential for NK cells commitment but also critical for NK cells homeostasis as well. We 
A

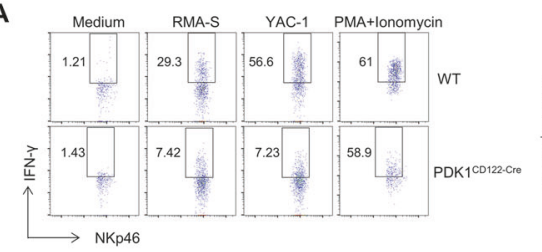

C

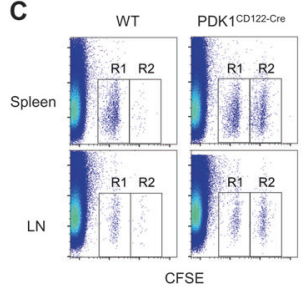

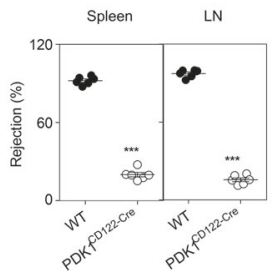

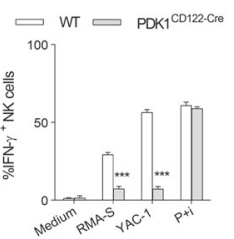

D

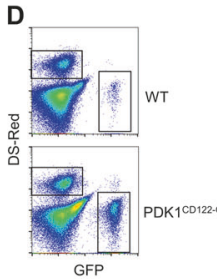

Fig. 2 CD122-Cre mediated $p d k 1$ deletion impairs NK cell-mediated immunosurveillance. a, b Intracellular staining of $\operatorname{IFN}-\gamma$ (a) and CD107a expression (b) on poly (i:c)-activated splenic NK cells. c Representative flow cytometry (left) and the percentage (Right) of $\beta 2 \mathrm{~m}$-deficient splenocytes rejection in WT and PDK1 ${ }^{\mathrm{CD} 122-\mathrm{Cre}}$ mice. R1, CFSE-low splenocytes from WT mice; and R2, CFSE-high splenocytes from $\beta 2 m$-deficient mice. d Representative flow cytometry (left) and the percentage (right) of RMA-S cell rejection $18 \mathrm{~h}$ after

previously reported that terminal deletion of PDK1 using a Ncr1-Cre line did not affect NK cell numbers, seemingly suggesting that PDK1 is not required for NK cells terminal differentiation and homeostasis. In view of the efficiency of deletion mediated by Ncr1-Cre in only some of the NK cells population (unpublished data), we decided to employ a new approach to re-address the question. We crossed $P D K 1^{f / f l} /$ $\mathrm{Ncrl}-\mathrm{Cre}^{+}$mice (hereafter referred to as PDK1 ${ }^{\mathrm{Ncrl}-\mathrm{Cre}}$ ) with Rosa26 $6^{\text {stop } Y F P}$ mice, in which the activity of the Cre enzyme is monitored by the expression of YFP. Notably, the wild-type $P d k l$ transcript was scarcely detectable in $\mathrm{YFP}^{+}$NK cells (Fig. 4a). We found that the percentage and number of YFP-labeled cells in PDK1 $1^{\mathrm{Ncrl}-\mathrm{Cre}}$ mice were moderately decreased (Fig. 4b, c). However, YFP-negative NK cells were increased, likely as a compensation for the absence of $\mathrm{YFP}^{+}$NK cells (Fig. 4d, e). The reduced NK cells percentage and number were observed in multiple organs and tissues (Fig. 4f, g). Consistently, we also revealed that the terminal deletion of PDK1 might also interrupt NK cells differentiation (Fig. 4h, i). Thus, we conclude that PDK1 is indeed required for terminal NK cells differentiation.

\section{PDK1 signaling is activated during NK cells activation following multiple types of stimulation}

The above data have clearly demonstrated the early stagespecific requirement for PDK1 in NK cells commitment and terminal differentiation. We next explored whether PDK1 signaling is involved in NK cells function. We first determined the activation status of PDK1 downstream

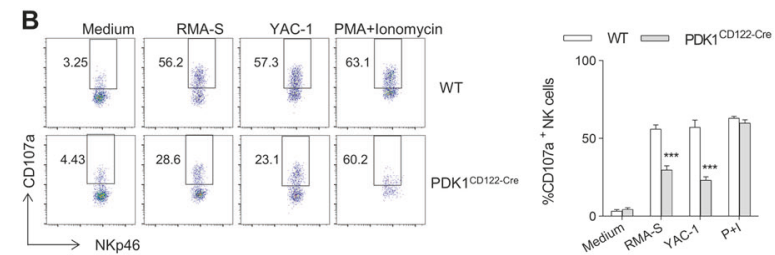

E
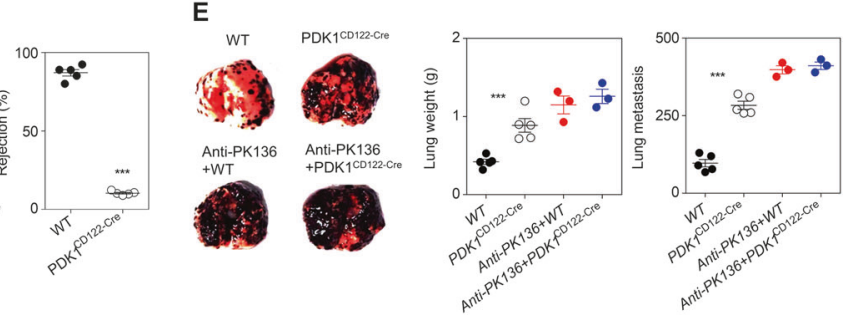

intraperitoneal injection of the indicated mice with a mixture of NK cell-sensitive RMA-S-GFP cells together with NK cell-resistant RMADsRed. e For the B16 metastasis assay, the indicated mice pretreated with or without antibody against NK1.1 were injected intravenously with $2 \times 10^{5}$ B16 cells. 14 days later, and the lung weights and numbers of tumor nodules were counted. Each symbol represents an individual mouse. The data represent one of two independent experiments, and values are expressed as the mean \pm s.d

signaling in NK cells from mice infected with MCMV or challenged with poly (I:C). We found that following MCMV infection or poly (I:C) stimulation the phosphorylation of $\mathrm{S}_{6}$ and $\mathrm{T}_{308}$ of AKT, were significantly decreased in PDK1-deleted NK cells (Fig. 5a, b). We next investigated whether the engagement of NK cells activating receptors might activate PDK1 signaling. To this end, NK cells were stimulated with plate-bound antibodies against NK1.1, which are ITAM-containing receptors. The cross-linking of NK1.1 enhanced the phosphorylation of S6 and $\mathrm{T}_{308}$ of AKT in WT NK cells, which was minimal in PDK1-null NK cells (Fig. 5c). The results were also confirmed by quantified Western blot (Fig. S2A). These findings suggest that multiple external stimuli, including virus-induced IFN $\alpha / \beta$ and the engagement of NK cells activating receptors, may trigger PDK1 signaling to facilitate NK cells activation.

\section{PDK1 promotes NK cells production of IFN- $\gamma$ by activating downstream $\mathbf{m T O R}$}

NK cells secrete IFN- $\gamma$ in response to either engagement of activating receptors with ligands on tumor target cells or priming with cytokines, such as IL-12 plus IL-18. Thus, we analyzed the critical role of PDK1 signaling in NK cells IFN- $\gamma$ production. First, NK cells were stimulated with hematopoietic tumor cells, RMA-S and YAC-1, or platecoated antibodies, respectively. Expectedly, a large proportion of NK cells could secrete IFN- $\gamma$ triggered by several types of stimuli, however, when PDK1 inhibitor, GSK2334470 was added, this activating effect was nearly 
A

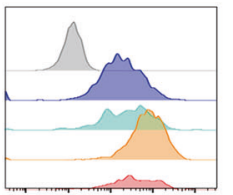

pS6

C

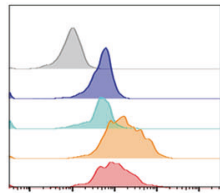

E4BP4

E

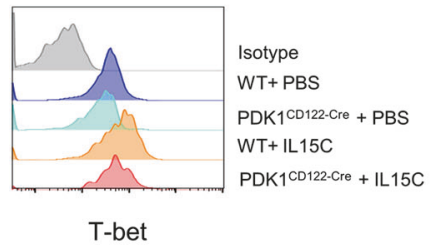

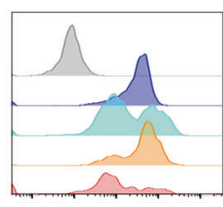

Eomes
B
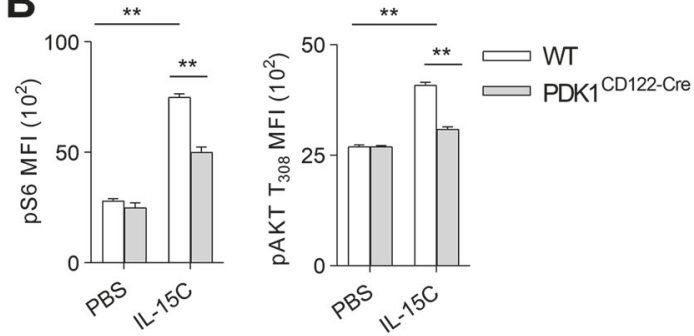

D
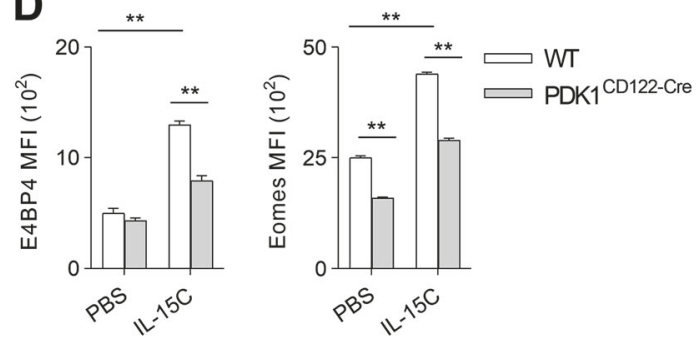

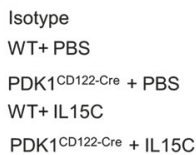

E

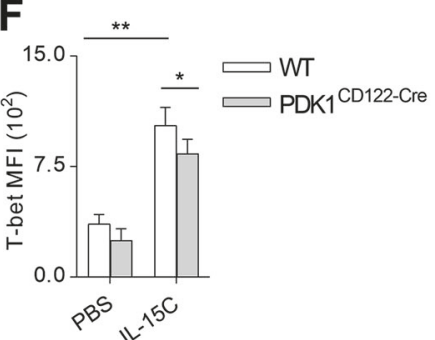

Fig. 3 PDK1 regulates NK cells commitment by inducing E4BP4. a, b The expression levels of phosphorylated $\mathrm{S} 6$ and $\mathrm{AKT} \mathrm{T}_{308}$ were detected with or without IL-15/IL-15Ra complexes (IL-15C) stimulation using flow cytometry (a) and the absolute MFI were quantified (b). c-f Representative overlaid histograms demonstrate E4BP4,
Eomes (c), and T-bet (e) expression level in WT and PDK1-deficient NK cells stimulated with or without IL-15C, and the absolute MFI was quantified (d, f). The data represent one of three independent experiments, and values are expressed as the mean \pm s.d abolished (Fig. 5d), which is indicative of the importance of PDK1 in NK cells IFN- $\gamma$ production. Moreover, PDK1 signaling likely acted through rewiring PI3Ks and mTOR, as evidenced by an assay in which the addition of Ly294002 or Torin1 to suppressing the activity of PI3Ks and mTOR, respectively, could also diminish the elicited effect (Fig. 5d). To confirm whether PDK1 regulation of $\mathrm{NK}$ cells function is mTOR-dependent, NK cells were pretreated with two mTOR inhibitors, rapamycin, and Torin. We found that the suppression of mTOR activity could significantly impair the ability of WT NK cells to secret IFN- $\gamma$, but this treatment almost had no effect on PDK1-deleted NK cells (Fig. S2B, C). Hence, PDK1 promotes NK cells IFN- $\gamma$ production likely by activating mTOR.

We then performed a genetic experiment to examine the effect of PDK1 deletion on NK cells priming by monitoring the expression levels of the early activation markers CD69 and CD25. The expression of these two receptors in NK cells from PDK $1^{\text {Ncr1-Cre }}$ mice exhibited a similar intensity of
CD69 and CD25 with those in WT NK cells (Fig. 5e), suggesting that PDK1 is not required for NK cells priming. Nevertheless, compared with WT NK cells, these normally primed PDK1-deficient NK cells failed to efficiently secrete IFN- $\gamma$ when they were stimulated with various stimuli (Fig. 5f-i). This dysfunction was not due to an overall defect of NK cells signaling, as these PDK1-deficient NK cells could produce comparable amounts of IFN- $\gamma$ when stimulated by PMA and ionomycin, which might bypass PDK1-mTOR signaling. Taken together, these data indicate that PDK1 is essential for NK cells IFN- $\gamma$ production.

\section{PDK1 promotes NK cells cytotoxicity against tumors by regulating the formation of NK-target conjugates and lytic synapses}

The upregulated expression of CD107a on the surface of NK cells correlates with NK cell-mediated lysis of target cells. We thus measured CD107a expression on NK cells following various types of stimulation. We found that compared with 

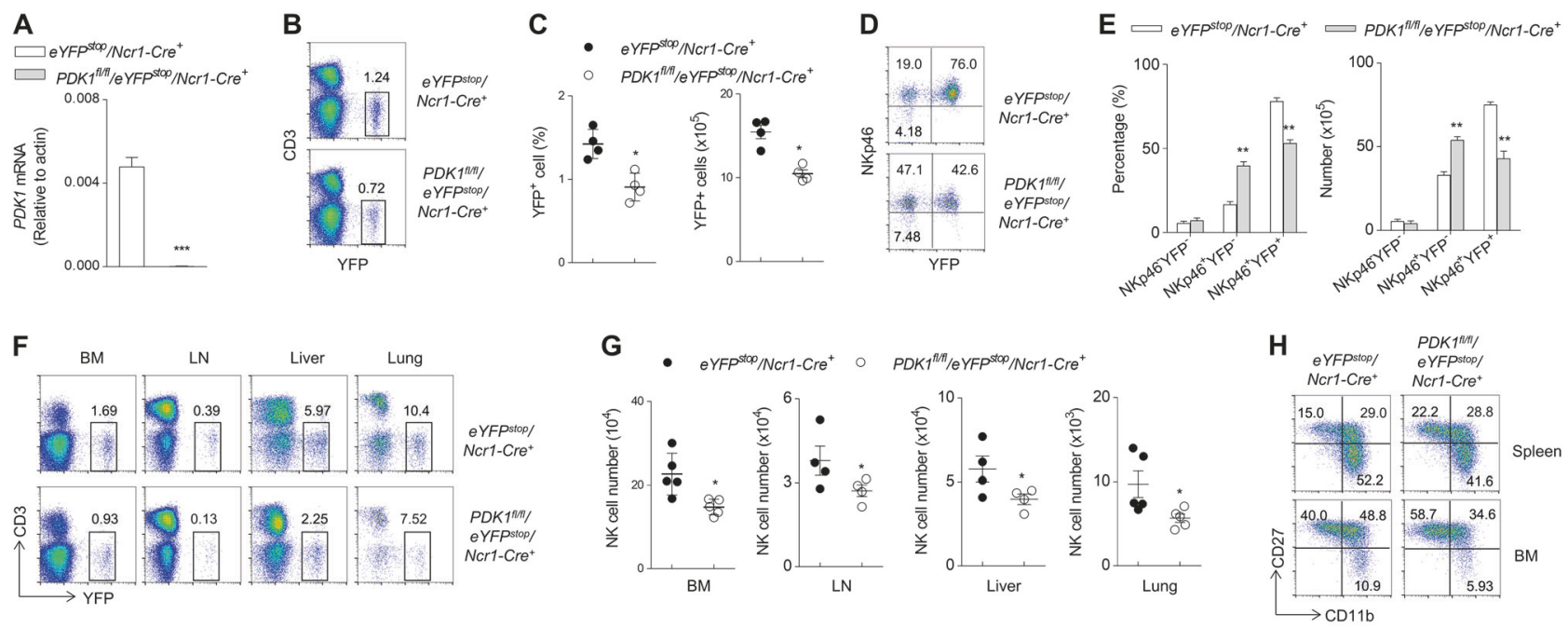

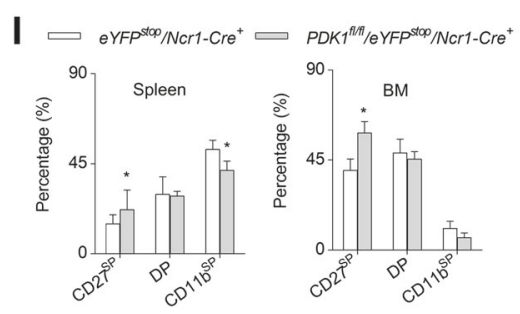

Fig. 4 PDK1 is required for terminal NK cells differentiation. a Quantitative reverse transcription-PCR analysis of $p d k 1$ expression in sorted $\mathrm{CD}^{-} \mathrm{NK} 1.1^{+} \mathrm{YFP}^{+}$cells from the indicated mice. b, $\mathbf{c}$ Representative flow cytometric plots (b), the percentage (c, left) and the absolute number (c, right) of $\mathrm{CD}^{-} \mathrm{YFP}^{+} \mathrm{NK}$ cells in the spleen of the indicated mice. d, e Representative flow cytometric plots (d), the percentage (e, left) and the absolute number (e, right) showing the expression of NKp46 and YFP in gated $\mathrm{CD}^{-} \mathrm{NK} 1.1^{+}$cells in the

WT NK cells, fewer NK cells from PDK1 $1^{\mathrm{Ncr} 1-\mathrm{Cre}}$ mice could up-regulate the expression of CD107a, granzyme $\mathrm{B}$ and perforin after co-culture with RMA-S or YAC-1 cells. Unexpectedly, NK cells from PDK1 $1^{\text {Ncrl-Cre }}$ mice exhibited a similar ability to express CD107a, granzyme B and perforin after stimulated with plate-coated antibodies against Ly49D or NK1.1 (Fig. 6a-d).

NK cells cytotoxicity relies heavily on integrindependent signaling, which helps NK cells to form stable synaptic interfaces with target cells. We thus made a hypothesis that the functional defect of PDK1-deficient NK cells in response to cellular targets and individual engagement of NK cells activating receptors might be due to the formation of conjugates between NK cells and target cells. Thus, we first determined whether PDK1deficient NK cells could stably contact target cells. The results showed that PDK1-deficient NK cells formed much fewer conjugates with RMA-S cells at the indicated time points (Fig. 6e, f). The formation of NK-target conjugates is the first step of the organization of the activating lytic synapse, which involves reorganization of the actin cytoskeleton and clustering of certain cell spleen of the indicated mice. $\mathbf{f}, \mathbf{g}$ Representative flow cytometric plots (f) and the absolute number (g) of $\mathrm{CD}^{-} \mathrm{YFP}^{+} \mathrm{NK}$ cells in the $\mathrm{BM}$, LNs, livers and lungs of the indicated mice. h CD27 and CD11b expression levels on $\mathrm{CD}^{-} \mathrm{YFP}^{+} \mathrm{NK}$ cells were detected by flow cytometry. i Percentage of cells in each developmental stage in gated $\mathrm{CD}^{-} \mathrm{YFP}^{+}$cells in the spleens and BM of WT and PDK $1^{\mathrm{Ncr} 1-\mathrm{Cre}}$ mice. The data represent one of three independent experiments, and values are expressed as the mean \pm s.d

surface receptors at the interface between NK cells and target cells. Thus, we next examined whether PDK1 deficiency might disrupt lytic synapse formation. When normal NK cells contacted RMA-S cells, F-actin polarization was visible at the interface of the majority of the conjugates. However, such actin aggregation was rarely observed in PDK1-deficient NK cells (Fig. 6g, h). In addition, we found that when WT NK cells were incubated with RMA-S cells, the adhesion molecule LFA-1 was polarized at the cell interface in the majority of the conjugates. Although PDK1-deficient NK cells did not have an expression level of lymphocyte functionassociated antigen 1 (LFA-1) (Fig. 6i), the loss of PDK1 in NK cells dramatically impaired LFA-1 polarization at the interface (Fig. 6j, k). To avoid the possibility that PDK1 deficiency interferes with the expression of certain adhesion molecules, the inhibitor GSK2334470 was used to transiently suppress PDK1 activity. In this model, GSK2334470-inhibited NK cells also failed to form stable and effective lytic synapses with targets (Fig. 61, m), which might result in less degranulation. Hence, NK cells degranulation requires PDK1 signaling. 
A
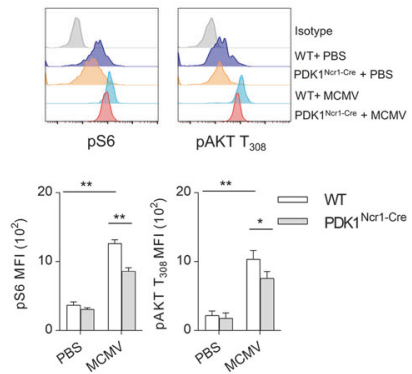

B
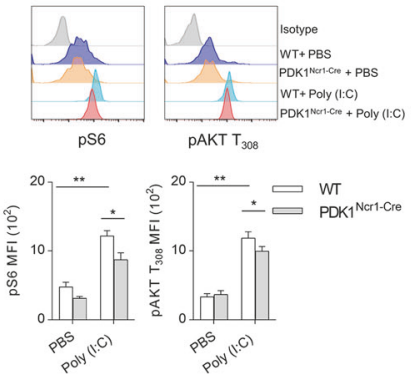

C
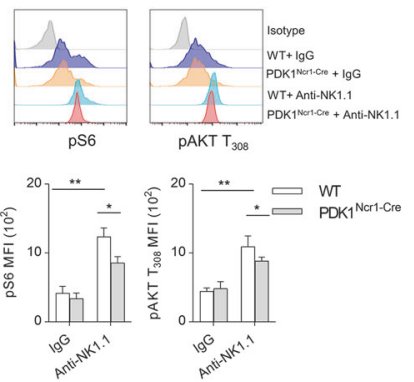
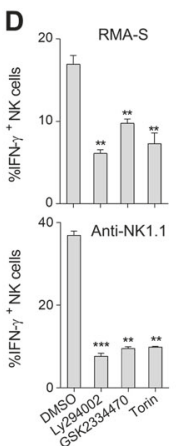
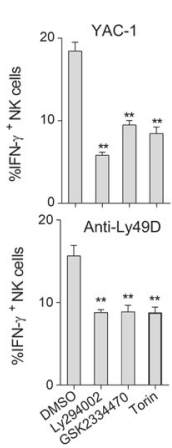

E

$\mathbf{F}$

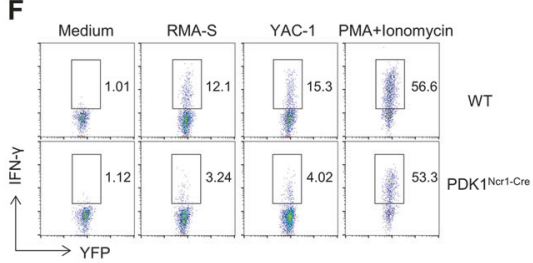

H

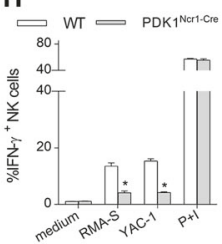

G

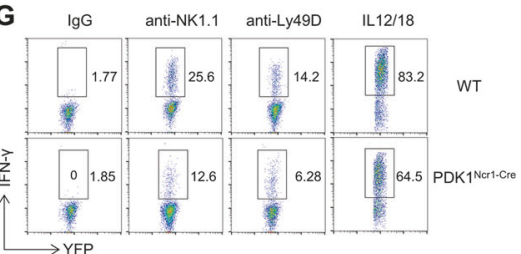

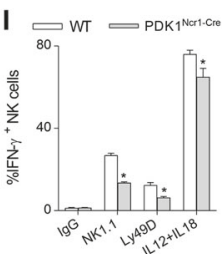

Fig. 5 PDK1-mTOR signaling plays an important role in NK cell IFN$\gamma$ production. a-c The expression levels of phosphorylated S6 and AKT $\mathrm{T}_{308}$ were detected by flow cytometry after splenic lymphocytes were stimulated with MCMV, poly (i:c), or anti-NK1.1 antibody (top) and the absolute MFI were quantified (lower). d Splenic lymphocytes were prepared from poly (i:c)-treated WT mice and pretreated with PI3K-mTOR signaling inhibitors. Intracellular staining was performed to assess the production of IFN- $\gamma$. The percentage quantification of
IFN- $\gamma$-positive NK cells is shown. e The expression levels of the early activation markers CD69 and CD25 were detected by flow cytometry after NK cells were stimulated with IL-15 or Poly(i:c) in the indicated mice. $\mathbf{f}-\mathbf{i}$ Representative flow cytometric profiles (f, $\mathbf{g})$ and the percentage $(\mathbf{h}, \mathbf{i})$ of IFN- $\gamma$ production in splenic NK cells from indicated mice following stimulation with tumor cells $(\mathbf{f}, \mathbf{h})$, plate-coated antibodies or cytokines $(\mathbf{g}, \mathbf{i})$. The data represent the mean \pm s.d and are representative of three independent experiments

\section{Terminal deletion of PDK1 impairs NK-mediated surveillance in vivo}

Next, we sought to examine NK cells effector function in PDK1 ${ }^{\text {Ncr1-Cre }}$ mice by transferring MHC-I-deficient hematopoietic cells into mice pretreated with poly (I:C). These MHC-I mismatched primary cells were rapidly eliminated by NK cells in WT mice, whereas NK cells ability to clearance $\beta 2 \mathrm{~m}^{-/-}$splenocytes was significantly

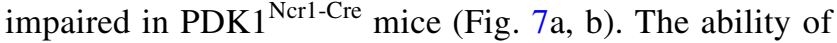
NK cells to eliminate RMA-S tumor cells was also compromised in this genotype (Fig. 7c, d). Finally, we measured the effect of PDK1 deletion on the ability of NK cells to prevent B16 melanoma metastasis, and we found that the lung weight and numbers of tumor colonies in PDK $1^{\text {Ncr1-Cre }}$ mice were moderately increased compared to those in WT mice (Fig. 7e, f). Thus, PDK1 deficiency impairs NK-mediated immunosurveillance towards "unwanted" cellular targets.

\section{Discussion}

IL-15 signaling is a rate-limiting step for NK cells development, and PI3K signaling plays an important role in this process [13, 14]. Our previous study found that PDK1 downstream of IL-15 signaling orchestrates NK cell development through activating mTOR; however, overactivation of mTOR is detrimental to NK cell development $[19,25]$. We employed three PDK1 conditional knockout mice in which the $p d k 1$ gene is deleted at three sequential stages, including HSC, NKp, and functional NK cells. Loss of PDK1 starting at the HSC stage caused NK cells developmental blockade at the NKp stage. NK cells commitment is characterized by the expression of CD122, the receptor subunit of IL-15. CD122-Cre was used to delete PDK1 at the NKp stage. Using this mouse model, we found that loss of PDK1 at the hematopoietic or progenitor stage showed comparable impairment of NK cells development, confirming that PDK1 is required for NK cells commitment. 

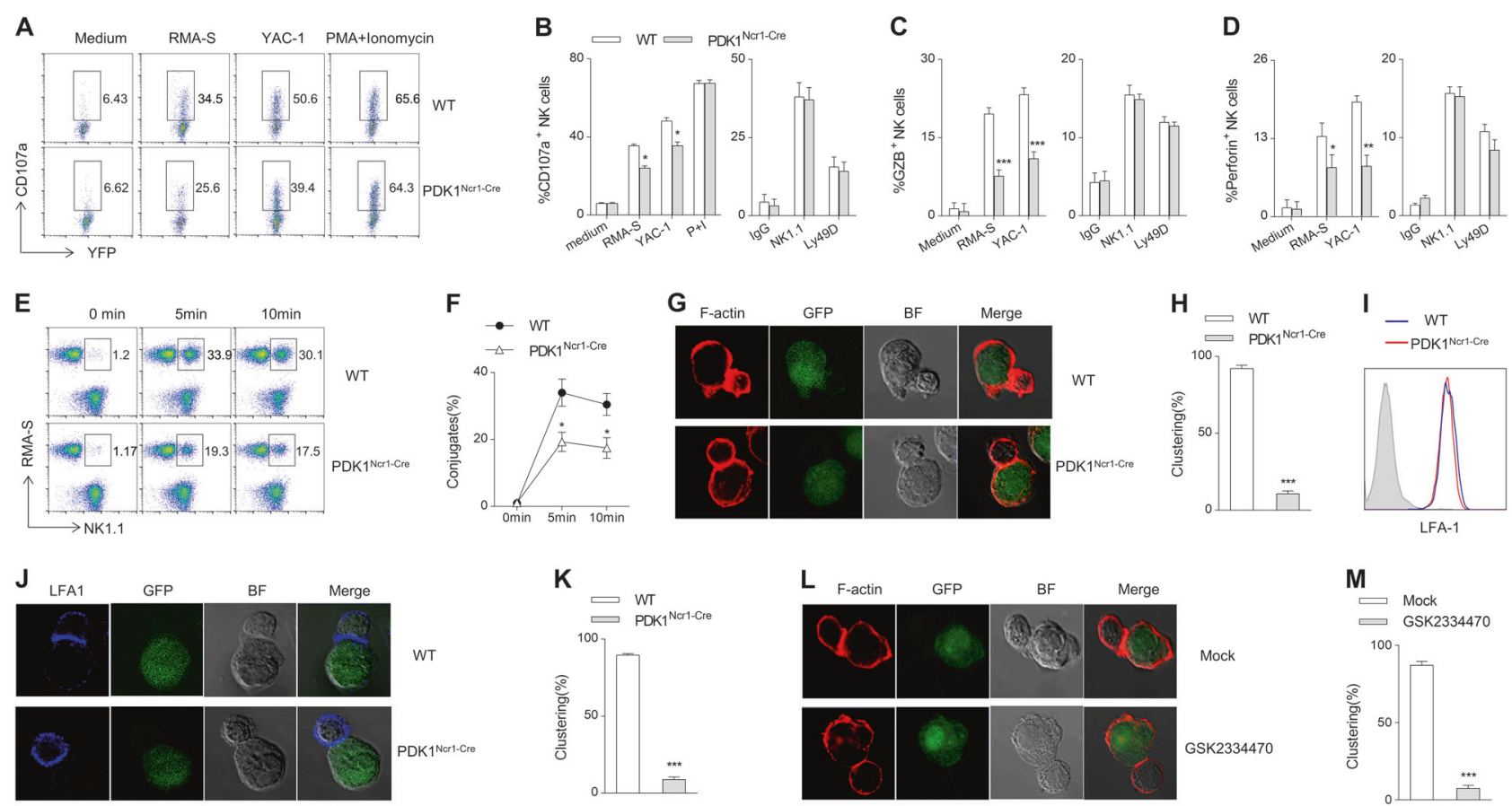

Fig. 6 PDK1 regulates NK cell conjugation with cellular targets. a-d Representative flow cytometric profiles (a) and the percentage of CD107a (b), granzyme B (c) and perforin (d) positive NK cells from indicated mice following stimulation with tumor cells or plate-coated antibodies. $\mathbf{e}, \mathbf{f}$ The percentage of NK-target cells conjugate formation from the indicated mice. $\mathbf{g}, \mathbf{j}$ NK cells from the indicated mice were mixed with RMA-S cells to analyze the localization of F-actin (g) or LFA-1 (j) in the conjugates. $\mathbf{h}, \mathbf{k}$ The data are expressed as the

percentages of conjugates with the polarized accumulation of F-actin (h) or LFA-1 (k). i The expression levels of LFA-1 in WT or PDK1deficient NK cells was detected by flow cytometry. I The expanded WT NK cells were pretreated with GSK2334470 and then mixed with RMA-S cells for $10 \mathrm{~min}$ to analyze the localization of F-actin in the conjugates. $\mathbf{m}$ The data are expressed as the percentage of conjugates with the polarized accumulation of F-actin. The data represent the mean \pm s.d and are representative of three independent experiments

Significant evidence has been found to indicate that transcription factors, including E4BP4, Eomes, and T-bet, play a critical role in NK cells development by preserving elevated levels of CD122 in NK cells [23, 24, 26]. Our previous studies have shown that loss of PDK1 in hematopoietic cells could inhibit IL-15-induced E4BP4 and Eomes expression in NK cells, thereby decreasing the expression level of CD122 [19]. In this study, we confirmed that PDK1 deficiency at the NKp stage inhibited the upregulation of E4BP4 and Eomes after IL-15 stimulation. Therefore, using a stage-specific gene-deficient mice model, we revealed the precise stages of NK cells development that are regulated by PDK1-mediated metabolic signaling.

Splenic NK cells can be induced to express high levels of granzyme B upon IL-15 stimulation. Notably, granzyme B induction was abrogated in the presence of inhibitors that block the PI3K-AKT-mTORC1 pathway [11]. Our results showed that activation of the PI3K-AKT-mTORC1 pathway is greatly increased in NK cells after stimulation with MCMV, Poly(I:C) or specific antibodies, indicating that the PI3K-mTOR pathway is involved in NK cell activation. We further used both pharmacological inhibition and genetic ablation to evaluate the importance of the PI3K-mTOR pathway in NK cell functions after exposure to various

stimuli. The treatment of several pharmacological inhibitors on NK cells during tumor cell stimulation resulted in severe defects in IFN- $\gamma$ production. Genetic ablation of PDK1 further demonstrated that PDK1 is an important regulator for NK cells activation. Loss of PDK1 inhibited IFN- $\gamma$ production by NK cells regardless of the type of stimulation. However, ablation of PDK1 only impaired the expression of CD107a as NK cells were stimulated with tumor cells but not upon antibody stimulation. It is thus suggested that PDK1 differentially regulates the two distinct effector functions of mature NK cells, and cytokine production by NK cells is highly dependent on PDK1 signaling.

In light of the dispensable role of PDK1-mediated signaling in NK-cell-activating receptor-triggered degranulation, there might be another mechanism whereby PDK1 regulates NK cell cytotoxicity upon exposure to tumor cells. During tumor cell stimulation, NK cells activating receptors could ligate with their ligands expressed on the tumor cells, inducing receptor clustering and actin reorganization at the synapse. An effective immunological synapse triggers the polarized movement of perforin to the interface [27]. Several lines of evidence have shown that PI3K signaling is involved in NK-target cell lytic synapse formation [28, 29]. 
A
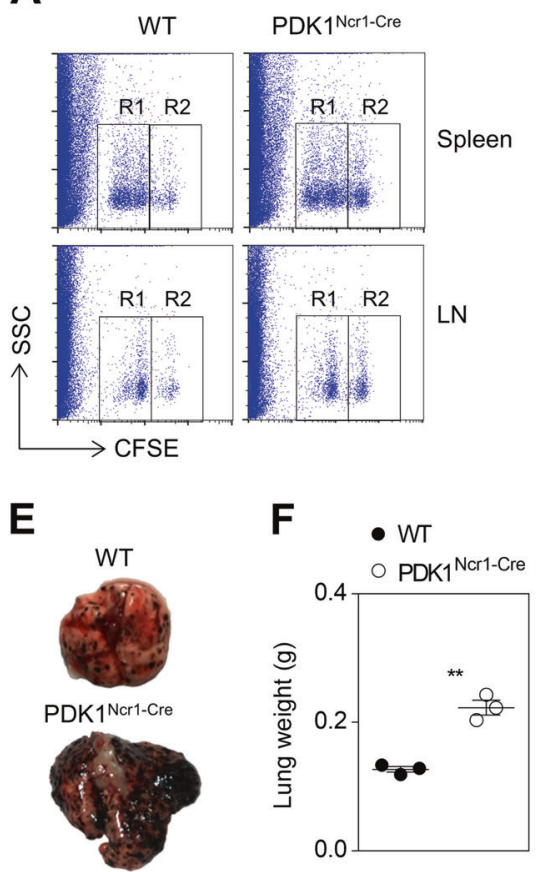

Fig. 7 Late-stage deficiency of PDK1 leads to reduced NK cellmediated immunosurveillance. NK cells in vivo functions, including MHC-II mismatched splenocyte rejection (a, b), RMA-S clearance

However, the pathway downstream of PI3Ks remained to be determined. In this study, we show that PDK1 is required for NK-target cell synapse formation and actin polymerization. Therefore, it seems likely that the impaired expression of CD107a in PDK1-deficient NK cells stimulated by tumor cells may be caused by the formation of ineffective synapses between NK cells and tumor cells.

In summary, we demonstrate the critical requirement of PDK1 for NKp cell commitment and its central role in connecting the PI3K-AKT-mTORC1 pathway during NK cell activation of effector function.

\section{Methods}

\section{Mice}

$P D K 1^{f l o x / f l o x}$ mice were a gift from Dario Alessi from the University of Dundee as previously described [19]. CD122-Cre and Ncr1-Cre mice were generated in our lab. $R a g 1^{-1-} \gamma \mathrm{c}^{-}$mice were described previously. $\beta 2 m$-deficient mice and C57BL/6 mice were purchased from Jackson Laboratory. Both female and male mice between 8 and 12 weeks of age were used in the experiments. All mice were maintained under specific pathogen-free animal facilities of Tsinghua University. All animal procedures were approved by the Animal Ethics Committee of Tsinghua University.
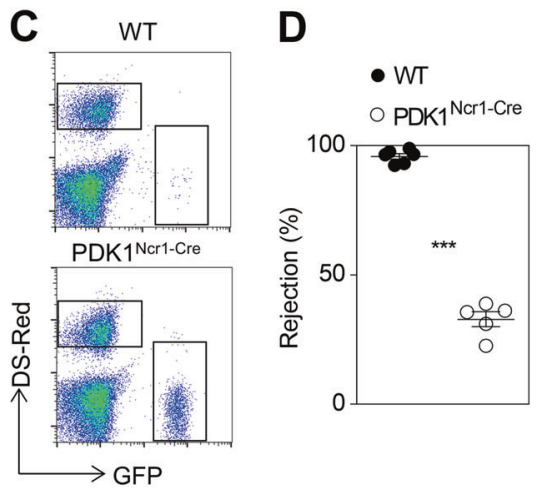

(c, d) and B16 melanoma lung metastasis (e, f), were analyzed as detailed for Fig. 2. All data represent at least three independent experiments, and calculated data are shown as the means \pm s.d

\section{Flow cytometry}

Flow cytometry was performed on a BD LSR II (BD Biosciences). Monoclonal antibodies against mouse CD3, NKp46, NK1.1, CD117, CD127, Ly49A, Ly49H, Ly49G2, CD11b, CD27, IFN- $\gamma$, CD107a, E4BP4, Eomes and isotype controls were purchased from eBioscience (San Diego, CA). Anti-Ly49C/I was purchased from BD Biosciences. Anti-phospho-S6 and anti-phospho-AKT $\left(\mathrm{T}_{308}\right)$ were obtained from Cell Signaling Technology (Beverly, MA). For analysis of surface markers, cells were incubated with indicated antibodies in PBS containing 2\% (wt/vol) FBS. The expression level was presented as percentage or net MFI, which was determined by subtracting mean fluorescence intensity of isotype control. For detection of phosphorylated signaling proteins, NK cells were fixed with Phosflow Lyse/Fix buffer, permeabilized with Phosflow Perm buffer III (BD), and then stained with antibodies.

\section{Detection of CD107a expression and intracellular staining for IFN- $\gamma$}

Mice were treated with intraperitoneal injection of poly I:C $(200 \mu \mathrm{g})$ for $18 \mathrm{~h}$. Poly I: C-activated splenocytes $\left(2 \times 10^{6}\right)$ were co-cultured with the same number of different target cells. For antibody stimulation, 24-well plates were precoated with indicated antibodies overnight. For cytokine stimulation, poly (I:C)-activated splenocytes were treated 
with cytokines (recombinant mouse IL-12 (10 ng/ml), recombinant mouse $\mathrm{IL}-18(10 \mathrm{ng} / \mathrm{ml}))$. BD GolgiStop ${ }^{\mathrm{TM}}$ reagent (BD Biosciences) was used to inhibit intracellular protein transport, and meanwhile, APC-conjugated antiCD107a antibody or respective control isotypes were added at the beginning of incubation. Splenocytes stimulated with PMA $(50 \mathrm{ng} / \mathrm{mL})$ plus ionomycin $(1 \mu \mathrm{M})$ were used as a positive control. Medium only was used as a negative control. $4 \mathrm{~h}$ after stimulation, cell mixtures were harvested for detection of intracellular IFN- $\gamma$ and CD107a expression.

\section{In vivo splenocyte rejection assay}

Splenocytes from WT or $\beta 2 \mathrm{~m}^{-/-}$mice were depleted of red blood cells by Ficoll-Hypaque density gradient centrifugation and then labeled with CFSE (Molecular Probes). Two types of CFSE-labeled splenocytes were mixed at a 1:1 ratio. A mixture of $2 \times 10^{6}$ splenocytes was intravenously injected into mice pre-treated with $200 \mu \mathrm{g}$ poly (I:C). $18 \mathrm{~h}$ later, CFSE-positive cells from the spleens and lymph nodes were determined by flow cytometry.

\section{In vivo RMA-S clearance assay}

Mice treated with $200 \mu \mathrm{g}$ Poly(I:C) for $18 \mathrm{~h}$ were intraperitoneally injected with a mixture of target cells, NKsensitive RMA-S cells expressing GFP $\left(10^{6}\right)$ and NK-nonsensitive RMA cells expressing Ds-Red $\left(10^{6}\right)$. Eighteen hours after tumor cell injection, the mice were sacrificed, and the cells in the peritoneal cavity were collected by repeated washing with PBS containing $2 \mu \mathrm{M}$ EDTA. The relative percentages of RMA-S and RMA cells were measured by flow cytometry.

\section{B16 melanoma lung metastasis mouse model}

B16F10 melanoma cells in the log phase were suspended in $1 \times$ HBSS and intravenously injected into mice $\left(2 \times 10^{5}\right.$ cells/mouse). Fourteen days later, the mice were sacrificed. The lungs were weighed, and the number of lung surface nodules was counted under a dissecting microscope.

\section{Conjugate formation assays}

NK cells were enriched from splenocytes using a positive selection kit (StemCell Technologies) and then cultured in RPMI 1640 medium containing 20\% fetal bovine serum, $\beta$ Mercapto alcohol and $1000 \mathrm{U} / \mathrm{ml}$ IL-2. 5 days later, NK cells were collected and stained with APC-conjugated antiMouse NK1.1 for $30 \mathrm{~min}$ on ice, Then the cells were washed and resuspended at $2 \times 10^{6}$ cells per $\mathrm{ml} .100 \mu \mathrm{l}$ cell suspension of NK cells and GFP-positive RMA-S cells were mixed together at a ratio of $1: 1$ and centrifuged at
$1500 \mathrm{rpm}\left(4^{\circ} \mathrm{C}\right)$ for $5 \mathrm{~min}$. After removing $150 \mu \mathrm{l}$ of supernatant, cells were stimulated for 0,5 and $10 \mathrm{~min}$ by incubation at $37^{\circ} \mathrm{C}$. Reactions were stopped by adding icecold RPMI 1640 medium and leaving cells on ice. The percentage of the $\mathrm{NK}_{1.1} 1^{+} \mathrm{GFP}^{+}$population was detected by flow cytometry.

\section{Immunofluorescence staining and confocal imaging}

NK cells $\left(1 \times 10^{5}\right)$ and GFP-positive RMA-S cells were mixed at a ratio of $1: 1$ and subjected to centrifugation for 7 $\min$ at $1000 \mathrm{rpm}\left(4^{\circ} \mathrm{C}\right)$. After removing $150 \mu \mathrm{l}$ of supernatant, the mixture were stimulated by incubation at $37^{\circ} \mathrm{C}$ for $10 \mathrm{~min}$, then fixed with $4 \%$ paraformaldehyde for $30 \mathrm{~min}$ at room temperature and blocked with PBS that containing $1 \%$ bovine serum albumin for $1 \mathrm{~h}$. The fixed cells were stained with rhodamine-labeled phalloidin (Invitrogen). Then, the cells were washed with PBS and mounted with Antifade Mounting Medium (Solarbio). The samples were analyzed with a laser scanning confocal microscope (LSM510 META; Carl Zeiss).

\section{Statistical analyses}

Unpaired Student's $t$-tests (two-tailed) were performed using the Prism software. A $p$ value of less than 0.05 was considered significant. $* p<0.05 ; * * p<0.01$; and $* * * p<$ 0.001 .

Acknowledgements Research reported in this publication was supported by the Natural Science Foundation of China (to ZD, 81725007, 31830027 and 31821003 , to MY, 81771666 and 81471523), Beijing Natural Science Foundation (5172018 to ZD), Guangzhou Science and Technology Project (to MY, 201707010395) and by 111 Project (B16201).

\section{Compliance with ethical standards}

Conflict of interest The authors declare that they have no conflict of interest.

Publisher's note: Springer Nature remains neutral with regard to jurisdictional claims in published maps and institutional affiliations.

\section{References}

1. Vivier E, Tomasello E, Baratin M, Walzer T, Ugolini S. Functions of natural killer cells. Nat Immunol. 2008;9:503-10.

2. Long EO, Kim HS, Liu D, Peterson ME, Rajagopalan S. Controlling natural killer cell responses: integration of signals for activation and inhibition. Annu Rev Immunol. 2013;31:227-58.

3. Karre K. Natural killer cell recognition of missing self. Nat Immunol. 2008;9:477-80.

4. Jandus C, Boligan KF, Chijioke O, Liu H, Dahlhaus M, Demoulins T, et al. Interactions between Siglec-7/9 receptors and ligands influence NK cell-dependent tumor immunosurveillance. J Clin Invest. 2014;124:1810-20. 
5. Raulet DH. Roles of the NKG2D immunoreceptor and its ligands. Nat Rev Immunol. 2003;3:781-90.

6. Lanier LL. Upon the tightrope: natural killer cell activation and inhibition. Nat Immunol. 2008;9:495-502.

7. Upshaw JL, Arneson LN, Schoon RA, Dick CJ, Billadeau DD, Leibson PJ. NKG2D-mediated signaling requires a DAP10-bound Grb2-Vav1 intermediate and phosphatidylinositol-3-kinase in human natural killer cells. Nat Immunol. 2006;7:524-32.

8. DiSanto JP, Muller W, Guy-Grand D, Fischer A, Rajewsky K. Lymphoid development in mice with a targeted deletion of the interleukin 2 receptor gamma chain. Proc Natl Acad Sci USA. 1995;92:377-81.

9. Sathe P, Delconte RB, Souza-Fonseca-Guimaraes F, Seillet C, Chopin M, Vandenberg CJ, et al. Innate immunodeficiency following genetic ablation of Mcl1 in natural killer cells. Nat Commun. 2014;5:4539.

10. Kennedy MK, Glaccum M, Brown SN, Butz EA, Viney JL, Embers M, et al. Reversible defects in natural killer and memory CD8 T cell lineages in interleukin 15-deficient mice. J Exp Med. 2000;191:771-80.

11. Nandagopal N, Ali AK, Komal AK, Lee SH. The critical role of IL-15-PI3K-mTOR pathway in natural killer cell effector functions. Front Immunol. 2014;5:187.

12. Donnelly RP, Loftus RM, Keating SE, Liou KT, Biron CA, Gardiner CM, et al. mTORC1-dependent metabolic reprogramming is a prerequisite for NK cell effector function. J Immunol. 2014; 193:4477-84.

13. Tassi I, Cella M, Gilfillan S, Turnbull I, Diacovo TG, Penninger $\mathrm{JM}$, et al. p110gamma and p110delta phosphoinositide 3-kinase signaling pathways synergize to control development and functions of murine NK cells. Immunity. 2007;27:214-27.

14. Guo H, Samarakoon A, Vanhaesebroeck B, Malarkannan S. The p110 delta of PI3K plays a critical role in NK cell terminal maturation and cytokine/chemokine generation. J Exp Med. 2008;205:2419-35.

15. Marcais A, Cherfils-Vicini J, Viant C, Degouve S, Viel S, Fenis A, et al. The metabolic checkpoint kinase mTOR is essential for IL-15 signaling during the development and activation of NK cells. Nat Immunol. 2014;15:749-57.

16. Finlay DK, Rosenzweig E, Sinclair LV, Feijoo-Carnero C, Hukelmann JL, Rolf J, et al. PDK1 regulation of mTOR and hypoxia-inducible factor 1 integrate metabolism and migration of CD8+T cells. J Exp Med. 2012;209:2441-53.
17. Hinton HJ, Alessi DR, Cantrell DA. The serine kinase phosphoinositide-dependent kinase 1 (PDK1) regulates $\mathrm{T}$ cell development. Nat Immunol. 2004;5:539-45.

18. Baracho GV, Cato MH, Zhu Z, Jaren OR, Hobeika E, Reth M, et al. PDK1 regulates B cell differentiation and homeostasis. Proc Natl Acad Sci USA. 2014;111:9573-8.

19. Yang M, Li D, Chang Z, Yang Z, Tian Z, Dong Z. PDK1 orchestrates early $\mathrm{NK}$ cell development through induction of E4BP4 expression and maintenance of IL-15 responsiveness. J ExpMed. 2015;212:253-65.

20. Yu J, Freud AG, Caligiuri MA. Location and cellular stages of natural killer cell development. Trends Immunol. 2013;34:57382.

21. Chiossone L, Chaix J, Fuseri N, Roth C, Vivier E, Walzer T. Maturation of mouse NK cells is a 4-stage developmental program. Blood. 2009;113:5488-96.

22. Robinette ML, Bando JK, Song W, Ulland TK, Gilfillan S, Colonna M. IL-15 sustains IL-7R-independent ILC2 and ILC3 development. Nat Commun. 2017;8:14601.

23. Kamizono S, Duncan GS, Seidel MG, Morimoto A, Hamada K, Grosveld G, et al. Nfil3/E4bp4 is required for the development and maturation of NK cells in vivo. J Exp Med. 2009;206:297786.

24. Gascoyne DM, Long E, Veiga-Fernandes H, de Boer J, Williams $\mathrm{O}$, Seddon B, et al. The basic leucine zipper transcription factor E4BP4 is essential for natural killer cell development. Nat Immunol. 2009;10:1118-24.

25. Yang M, Chen S, Du J, He J, Wang Y, Li Z, et al. NK cell development requires Tsc1-dependent negative regulation of IL15-triggered mTORC1 activation. Nat Commun. 2016;7:12730.

26. Intlekofer AM, Takemoto N, Wherry EJ, Longworth SA, Northrup JT, Palanivel VR, et al. Effector and memory CD8 + T cell fate coupled by T-bet and eomesodermin. Nat Immunol. 2005;6:1236-44.

27. Orange JS. Formation and function of the lytic NK-cell immunological synapse. Nat Rev Immunol. 2008;8:713-25.

28. Jiang K, Zhong B, Gilvary DL, Corliss BC, Hong-Geller E, Wei $\mathrm{S}$, et al. Pivotal role of phosphoinositide-3 kinase in regulation of cytotoxicity in natural killer cells. Nat Immunol. 2000;1:419-25.

29. Giurisato E, Cella M, Takai T, Kurosaki T, Feng Y, Longmore GD, et al. Phosphatidylinositol 3-kinase activation is required to form the NKG2D immunological synapse. Mol Cell Biol. 2007;27:8583-99. 